\title{
Discovery of a Second Transient Low-mass X-ray Binary in the Globular Cluster Ngc 6440
}

\section{Citation}

Heinke, C. O., D. Altamirano, H. N. Cohn, P. M. Lugger, S. A. Budac, M. Servillat, M. Linares, et al. 2010. "DISCOVERY OF A SECOND TRANSIENT LOW-MASS X-RAY BINARY IN THE GLOBULAR CLUSTER NGC 6440." The Astrophysical Journal 714 (1): 894-903. https:// doi.org/10.1088/0004-637x/714/1/894.

\section{Permanent link}

http://nrs.harvard.edu/urn-3:HUL.InstRepos:41399755

\section{Terms of Use}

This article was downloaded from Harvard University's DASH repository, and is made available under the terms and conditions applicable to Other Posted Material, as set forth at http:// nrs.harvard.edu/urn-3:HUL.InstRepos:dash.current.terms-of-use\#LAA

\section{Share Your Story}

The Harvard community has made this article openly available.

Please share how this access benefits you. Submit a story.

Accessibility 


\title{
DISCOVERY OF A SECOND TRANSIENT LOW-MASS X-RAY BINARY IN THE GLOBULAR CLUSTER NGC 6440
}

\author{
C. O. Heinke ${ }^{1}$, D. Altamirano ${ }^{2}$, H. N. Cohn $^{3}$, P. M. Lugger ${ }^{3}$, S. A. Budac ${ }^{1}$, M. Servillat ${ }^{4}$, M. Linares ${ }^{2,5}$, \\ T. E. Strohmayer ${ }^{6}$, C. B. MarkwardT ${ }^{6}$, R. Wijnands ${ }^{2}$, J. H. Swank ${ }^{6}$, C. KNigge ${ }^{7}$, C. Bailyn ${ }^{8}$, And J. E. Grindlay ${ }^{4}$ \\ ${ }^{1}$ Department of Physics, University of Alberta, Room 238 CEB, Edmonton, AB T6G 2G7, Canada; heinke@ ualberta.ca

\begin{abstract}
We have discovered a new transient low-mass X-ray binary, NGC $6440 \mathrm{X}-2$, with Chandra/ACIS, RXTE/PCA, and Swift/XRT observations of the globular cluster NGC 6440. The discovery outburst (2009 July 28-31) peaked at $L_{X} \sim 1.5 \times 10^{36} \mathrm{erg} \mathrm{s}^{-1}$ and lasted for $<4$ days above $L_{X}=10^{35} \mathrm{erg} \mathrm{s}^{-1}$. Four other outbursts (2009 May 29-June 4, August 29-September 1, October 1-3, and October 28-31) have been observed with RXTE/PCA (identifying millisecond pulsations) and Swift/XRT (confirming a positional association with NGC 6440 X-2), with similar peak luminosities and decay times. Optical and infrared imaging did not detect a clear counterpart, with best limits of $V>21, B>22$ in quiescence from archival Hubble Space Telescope imaging, $g^{\prime}>22$ during the August outburst from Gemini-South GMOS imaging, and $J \gtrsim 18.5$ and $K \gtrsim 17$ during the July outburst from CTIO $4 \mathrm{~m}$ ISPI imaging. Archival Chandra X-ray images of the core do not detect the quiescent counterpart $\left(L_{X}<(1-2) \times 10^{31} \mathrm{erg} \mathrm{s}^{-1}\right)$ and place a bolometric luminosity limit of $L_{\mathrm{NS}}<6 \times 10^{31} \mathrm{erg} \mathrm{s}^{-1}$ (one of the lowest measured) for a hydrogen atmosphere neutron star. A short Chandra observation 10 days into quiescence found two photons at NGC 6440 X-2's position, suggesting enhanced quiescent emission at $L_{X} \sim 6 \times 10^{31} \mathrm{erg} \mathrm{s}^{-1}$. NGC $6440 \mathrm{X}-2$ currently shows the shortest recurrence time $(\sim 31$ days $)$ of any known X-ray transient, although regular outbursts were not visible in the bulge scans before early 2009. Fast, low-luminosity transients like NGC 6440 X-2 may be easily missed by current X-ray monitoring.
\end{abstract}

Key words: dense matter - pulsars: general - stars: neutron - X-rays: binaries

Online-only material: color figures

\section{INTRODUCTION}

The dense cores of globular clusters are known to be efficient factories for dynamically producing tight binaries containing heavy stars and thus X-ray binaries (Clark 1975; Hut et al. 1991; Pooley et al. 2003). Thirteen luminous $\left(L_{X}>10^{35} \mathrm{erg} \mathrm{s}^{-1}\right)$ lowmass X-ray binaries (LMXBs) have previously been identified in Galactic globular clusters (see Verbunt \& Lewin 2006), concentrated in the densest and most massive clusters (Verbunt \& Hut 1987; Verbunt 2003). Luminous LMXBs in globular clusters of other galaxies are clearly concentrated in the most massive, most metal-rich, and densest globular clusters (Kundu et al. 2002; Sarazin et al. 2003; Jordán et al. 2004). A critical question for studies of globular cluster LMXBs is whether $\mathrm{X}$-ray emission from a globular cluster is due to one LMXB or multiple LMXBs. This affects the inferred nature of sources (Dotani et al. 1990; Di Stefano et al. 2002; Maccarone et al. 2007) and luminosity functions (Sivakoff et al. 2007). For example, an apparent contradiction in the qualities of the LMXB in M15 was resolved by the identification of two persistent X-ray sources in the cluster (White \& Angelini 2001). Identification with Chandra of multiple quiescent LMXBs in several globular clusters (Grindlay et al. 2001; Pooley et al. 2002; Heinke et al. 2003) has suggested that transient LMXB outbursts from a cluster might arise from different sources, although some LMXBs possess distinctive characteristics (e.g., the Rapid Burster; Homer et al. 2001). Here, we report the discovery and outburst monitoring of the 14th luminous LMXB in a Galactic globular cluster, NGC 6440, the first cluster to show two luminous transient LMXBs (both of which show millisecond pulsations). In a companion paper, Altamirano et al. (2010a) identify this LMXB as a new ultracompact accreting millisecond X-ray pulsar (AMXP), after discovering coherent $206 \mathrm{~Hz}$ pulsations (Altamirano et al. 2009) and fitting the frequency drift to a 57.3-minute orbital period.

NGC 6440 is a globular cluster near the Galactic center, at a distance of $8.5 \mathrm{kpc}$ (Ortolani et al. 1994), with $N_{H}=$ $5.9 \times 10^{21} \mathrm{~cm}^{-2}$ (Harris 1996). A luminous X-ray source (MX 1746-20) was identified in this cluster in 1971 (Markert et al. 1975), and the cluster was detected at a much lower flux level in 1980 (Hertz \& Grindlay 1983), identified with quiescent emission from the luminous X-ray source. An X-ray source in the cluster (SAX J1748.9-2021) was observed in outburst again in 1998 (in’t Zand et al. 1999), 2001 (in't Zand et al. 2001), and 2005 (Markwardt \& Swank 2005). Pooley et al. (2002) observed the cluster with Chandra in 2000, identifying 24 sources within two core radii of the cluster. One (CX1) was positionally identified with a blue variable optical counterpart during the 1998 outburst (Verbunt et al. 2000) and with the outbursting LMXB in 2001 (in't Zand et al. 2001). Altamirano et al. (2008) identified intermittent $442 \mathrm{~Hz}$ pulsations in both the 2001 and 2005 outbursts (the latter also identified by Gavriil 

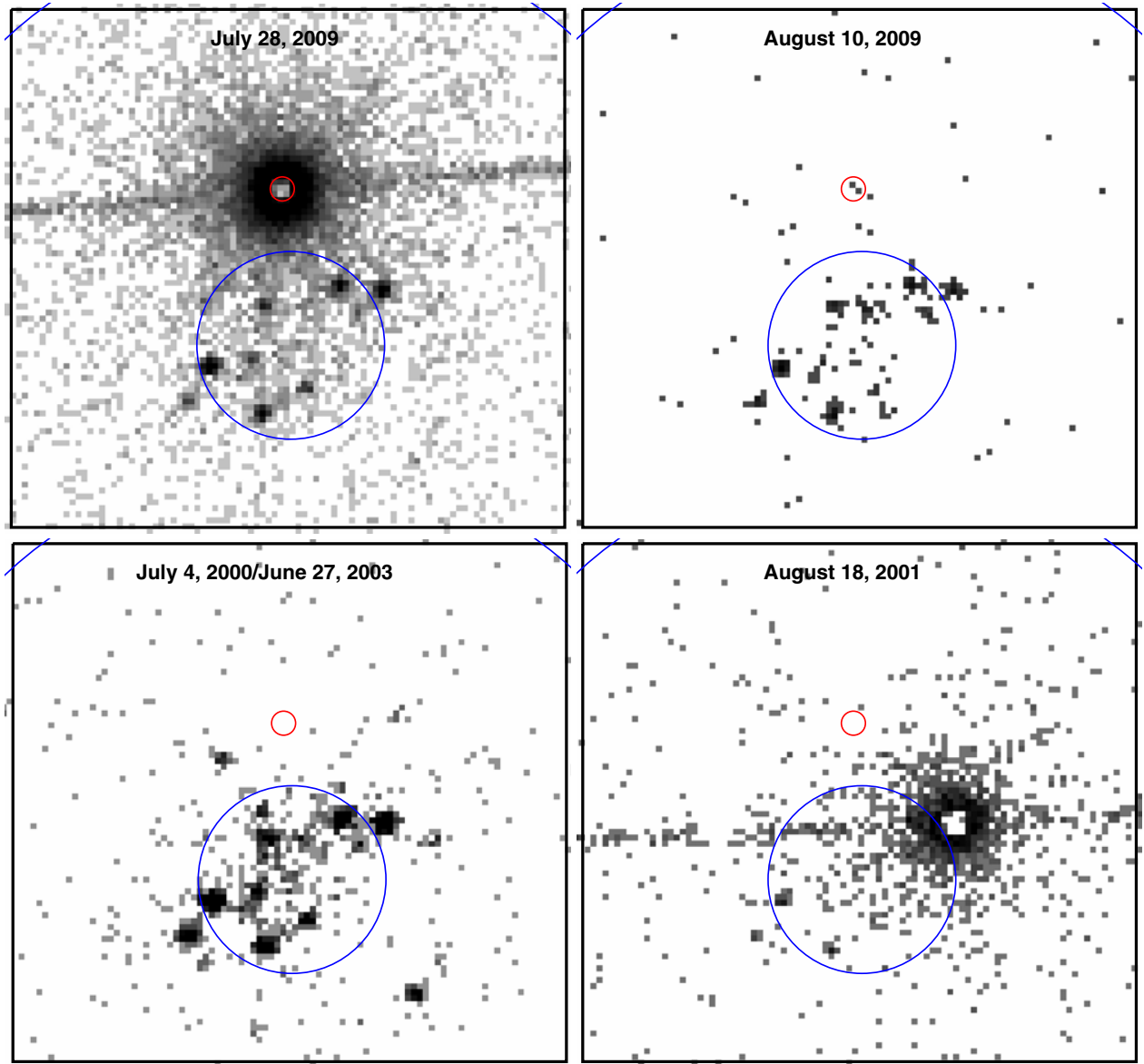

Figure 1. Chandra/ACIS-S images of NGC 6440 during the 2009 July outburst of NGC $6440 \mathrm{X}-2$ (upper left, $0.5-2.5 \mathrm{keV}$ ) and 13 days later (upper right, $0.3-7 \mathrm{keV}$ ). Below, we show a merged image from quiescent epochs in 2000 and 2003 (lower left, $0.3-7 \mathrm{keV}$ ) and an image from the 2001 outburst (lower right, $0.3-7 \mathrm{keV}$ ). The positions of NGC 6440 X-2 (red circle, $\left.1^{\prime \prime}\right)$ and the cluster core (0.13) and half-mass (0.58) radii (large circles) are indicated. North is up, east to the left.

(A color version of this figure is available in the online journal.)

et al. 2007). Thus, CX1 can be confidently held responsible for the 1998, 2001, and 2005 outbursts. We here identify a second transient luminous LMXB in NGC 6440 and confidently identify five outbursts from this transient during 2009 using its source position. Preliminary results were presented in Heinke \& Budac (2009) and Heinke et al. (2009a, 2009c); these results supersede those.

\section{X-RAY ANALYSIS}

\subsection{Chandra in Outburst}

NGC 6440 was observed with the Chandra ACIS-S detector for $49.1 \mathrm{ks}$, from 2009 July 28, 15:16 (TT) to 2009 July 29, $05: 15$, using a $1 / 2$ subarray. We searched for periods of elevated background, but found none. We used the level-2 event files provided by the CXC and CIAO $4.1^{9}$ for our analysis. Images were produced in the $0.3-7 \mathrm{keV}$ and $0.5-2.5 \mathrm{keV}$ bands, both of which are dominated by the scattered halo (a combination of dust grain scattering and the intrinsic point-spread function of the Chandra mirrors) and readout streak from a bright transient LMXB, heavily affected by pileup. ${ }^{10}$ Several faint point sources are clearly visible, which can be confidently identified with the cluster X-ray sources identified by Pooley et al. (2002). In Figure 1, we show Chandra images of NGC 6440 during the

\footnotetext{
9 http://cxc.harvard.edu/ciao

10 See the Chandra Proposer's Observatory Guide, chapter 6.
}

2009 outburst, shortly after the 2009 outburst, during quiescent observations in 2000-2003 (Pooley et al. 2002; Cackett et al. 2005) and during the 2001 outburst (in't Zand et al. 2001). Clearly this is a new transient.

The CIAO detection algorithm wavdetect was run on a $0.3-7 \mathrm{keV}$ image of the cluster core to identify the positions of known cluster sources, which we shift $(\Delta \mathrm{R} . \mathrm{A} .=+0.008 \mathrm{~s}$, $\Delta$ decl. $=+0^{\prime}$ '31) to align with the (ICRS) astrometry of Pooley et al. (2002). We estimated (by eye) the center of the symmetric "hole" in the counts by matching circles to the doughnutshaped locus of maximum count rate in the LMXB halo, in both wavebands. Our result is $(\mathrm{J} 2000)$ R.A. $=17: 48: 52.76(2)$, decl. $=$ $-20: 21: 24.0(1)$ (1 $\sigma$ values, after our astrometric correction), giving it the IAU name CXOGlb J174852.7-202124 and (for shorthand) NGC 6440 X-2. Detailed analysis of the remaining cluster sources will be presented elsewhere.

To measure the spectrum and luminosity of the transient, we extracted a spectrum from the readout streak, excluding a $20^{\prime \prime}$ radius circle around the piled-up transient. We extracted background from rectangular regions above and below the readout streak, computed response functions for the position of the transient, and corrected the exposure time of the spectrum. We binned the spectrum to 60 counts per bin to improve its statistics, and excluded data over $8 \mathrm{keV}$ and below $0.5 \mathrm{keV}$. An absorbed power law fits the data (Table 1, Figure 2), with photon index $1.7 \pm 0.1$. We found no evidence for a 6.4 or $6.7 \mathrm{keV}$ iron line, with a $90 \%$ upper limit of $0.4 \mathrm{keV}$ on its equivalent width. 
Table 1

Spectral Fits to NGC 6440 X-2

\begin{tabular}{|c|c|c|c|c|c|c|c|}
\hline $\begin{array}{c}\text { Start Time } \\
(2009)\end{array}$ & Instrument & $\begin{array}{c}\text { Exposure } \\
(\mathrm{ks})\end{array}$ & $\begin{array}{c}N_{H} \\
\left(\times 10^{21} \mathrm{~cm}^{-2}\right) \\
\end{array}$ & $\Gamma$ & $\chi_{v}^{2} /$ dof & $\begin{array}{c}L_{X}, 0.5-10 \mathrm{keV} \\
\left(\operatorname{erg~s}^{-1}\right)\end{array}$ & ObsID \\
\hline Jun 4, 01:04 & Swift/XRT & 1.1 & $0.59^{\mathrm{a}}$ & $2.4 \pm 0.5$ & $49^{b}$ & $3.3 \pm 0.8 \times 10^{34}$ & 00031421001 \\
\hline Jun $11,17: 32$ & Swift/XRT & 1.9 & $0.59^{\mathrm{a}}$ & $4.2_{-1.1}^{+1.2}$ & $59^{\mathrm{b}}$ & $1.0_{-0.5}^{+1.0} \times 10^{34}$ & 00031421002 \\
\hline Jul 28, 20:17 & Chandra/ACIS-S & 49.1 & $0.69 \pm 0.06$ & $1.79 \pm 0.10$ & $0.98 / 59$ & $1.55 \pm 0.06 \times 10^{36}$ & 10060 \\
\hline Jul 30, 18:47 & $R X T E / \mathrm{PCA}$ & 1.9 & $0.59^{\mathrm{a}}$ & $2.1_{-0.7}^{+0.8}$ & $0.57 / 27$ & $9.5_{-4.5}^{+13} \times 10^{34}$ & 94044-04-01-00 \\
\hline Jul 31, 04:55 & Swift/XRT & 1.8 & $0.59^{\mathrm{a}}$ & $2.3 \pm 0.4$ & $92^{\mathrm{b}}$ & $3.1 \pm 0.6 \times 10^{34}$ & 00031421003 \\
\hline Aug 4, 16:26 & Swift/XRT & 1.0 & $0.59^{\mathrm{a}}$ & $2.9_{-1.1}^{+1.2}$ & $37^{\mathrm{b}}$ & $7_{-3}^{+8} \times 10^{33}$ & 00031421004 \\
\hline Aug 6, 01:50 & Swift/XRT & 1.9 & $0.59^{\mathrm{a}}$ & $3.4_{-1.0}^{+1.2}$ & $99^{\mathrm{b}}$ & $1.1_{-0.5}^{+0.9} \times 10^{34}$ & 00031421005 \\
\hline Aug $10,13: 28$ & Swift/XRT & 0.5 & $0.59^{\mathrm{a}}$ & $\mathrm{c}$ & $\cdots$ & $7_{-5}^{+8} \times 10^{33}$ & 00031421007 \\
\hline Aug 10, 08:10 & Chandra/ACIS-S & 4.9 & $0.59^{\mathrm{a}}$ & $\mathrm{c}$ & $\cdots$ & $6_{-5}^{+9} \times 10^{31}$ & 11802 \\
\hline Aug $30,1: 31^{\mathrm{d}}$ & $R X T E / \mathrm{PCA}$ & 3.2 & $0.59^{\mathrm{a}}$ & $1.79 \pm 0.02$ & $0.84 / 54$ & $2.55 \pm 0.05 \times 10^{36}$ & $94044-04-02-00$ \\
\hline Sep $1,3: 43^{d}$ & $R X T E / \mathrm{PCA}$ & 14.1 & $0.59^{\mathrm{a}}$ & $1.5_{-0.8}^{+0.8}$ & $0.8 / 33$ & $<4 \times 10^{34}$ & 94044-04-02-01 \\
\hline Sep 1, 4:33 & Swift/XRT & 1.0 & $0.59^{\mathrm{a}}$ & $3.8_{-0.6}^{+0.7}$ & $90^{\mathrm{b}}$ & $6 \pm 2 \times 10^{34}$ & 00031421009 \\
\hline Sep 2, 13:01 & Swift/XRT & 0.4 & $0.59^{\mathrm{a}}$ & c & $\cdots$ & $1.2_{-6}^{+1.1} \times 10^{34}$ & 00031421010 \\
\hline Sep 4, 06:49 & Swift/XRT & 0.4 & $0.59^{\mathrm{a}}$ & c & $\cdots$ & $1.5_{-0.7}^{+1.3} \times 10^{34}$ & 00031421012 \\
\hline Oct $1,08: 47$ & Swift/XRT & 0.7 & $0.59^{\mathrm{a}}$ & $1.79 \pm 0.19$ & $1.0 / 16$ & $5.9 \pm 0.6 \times 10^{35}$ & 00031421014 \\
\hline Oct $1,21: 58$ & $R X T E / \mathrm{PCA}$ & 1.5 & $0.59^{\mathrm{a}}$ & $1.8_{-0.2}^{+0.1}$ & $0.49 / 29$ & $4.5_{-0.4}^{+0.4} \times 10^{35}$ & 94044-04-03-00 \\
\hline Oct $2,20: 24$ & Swift/XRT & 1.0 & $0.59^{\mathrm{a}}$ & $1.9 \pm 0.3$ & $0.35 / 6$ & $1.0_{-0.1}^{+0.2} \times 10^{35}$ & 00031421015 \\
\hline Oct 3, 08:12 & $R X T E / \mathrm{PCA}$ & 3.2 & $0.59^{\mathrm{a}}$ & $\ldots$ & $1.1 / 28$ & $<3 \times 10^{34}$ & 94044-04-04-00 \\
\hline Oct $4,10: 46$ & Swift/XRT & 0.6 & $0.59^{\mathrm{a}}$ & $\mathrm{c}$ & $\ldots$ & $6_{-4}^{+6} \times 10^{33}$ & 00031421017 \\
\hline Oct $5,18: 59$ & Swift/XRT & 0.6 & $0.59^{\mathrm{a}}$ & $\mathrm{c}$ & $\cdots$ & $7_{-4}^{+7} \times 10^{33}$ & 00031421016 \\
\hline Oct $28,22: 29$ & $R X T E / \mathrm{PCA}$ & 0.9 & $0.59^{\mathrm{a}}$ & $1.8_{-0.1}^{+0.1}$ & $0.34 / 39$ & $8.6_{-0.8}^{+0.9} \times 10^{35}$ & $94315-01-04-01$ \\
\hline Oct $29,00: 19$ & Swift/XRT & 1.2 & $0.59^{\mathrm{a}}$ & $1.7_{-0.1}^{+0.1}$ & $1.03 / 40$ & $5.5_{-0.3}^{+0.3} \times 10^{35}$ & 00031421018 \\
\hline Oct $29,20: 25$ & $R X T E / \mathrm{PCA}$ & 0.9 & $0.59^{\mathrm{a}}$ & $2.2_{-0.5}^{+0.6}$ & $0.58 / 27$ & $2.1_{-0.8}^{+2.2} \times 10^{35}$ & $94315-01-04-02$ \\
\hline Oct $30,13: 28$ & Swift/XRT & 0.9 & $0.59^{\mathrm{a}}$ & $2.3_{-0.4}^{+0.3}$ & $1.15 / 8$ & $8.6_{-1.4}^{+1.4} \times 10^{34}$ & 00031421019 \\
\hline Oct $30,19: 58$ & $R X T E / \mathrm{PCA}$ & 0.8 & $0.59^{\mathrm{a}}$ & $3.0_{-2.3}^{+4.4}$ & $0.52 / 15$ & $5 \pm 4 \times 10^{34}$ & $94315-01-03-01$ \\
\hline Oct $31,13: 33$ & Swift/XRT & 0.9 & $\cdots$ & c & $\ldots$ & $1.2_{-0.4}^{+0.6} \times 10^{34}$ & 00031421020 \\
\hline Oct $31,17: 48$ & $R X T E / \mathrm{PCA}$ & 1.1 & $0.59^{\mathrm{a}}$ & (2) & $0.58 / 23$ & $<3.1 \times 10^{34}$ & $94315-01-03-02$ \\
\hline Nov 1, 13:39 & Swift/XRT & 0.9 & $\cdots$ & $\mathrm{c}$ & $\ldots$ & $6_{-3}^{+4} \times 10^{33}$ & 00031421021 \\
\hline Nov 2, 13:45 & Swift/XRT & 0.9 & $\cdots$ & c & $\cdots$ & $4_{-2}^{+4} \times 10^{33}$ & 00031421022 \\
\hline
\end{tabular}

Notes. Absorbed power-law spectral fits, using distance of $8.5 \mathrm{kpc}$. Errors are $90 \%$ confidence for a single parameter. Swift results apply to flux from entire cluster (see the text for details), while $R X T E$ results subtract the quiescent $R X T E / \mathrm{PCA}$ fitted spectrum (inferred $L_{X}=4 \times 10^{35}$ ergs s ${ }^{-1}$ ) produced by the Galactic Ridge. After an $R X T E / P C A$ observation finds only an upper limit during the decline of an outbursts, we omit the later RXTE/PCA observations from this table (omitted ObsIDs are 94044-04-02-13, 94044-04-02-02, 94044-04-02-03, 94044-05-01-00, 94315-01-03-03, 94315-01-03-04, 94315-01-03-09, 94315-01-03-05, 94315-01-03-06, 94315-01-03-00, and 94315-01-03-07).

${ }^{\text {a }} N_{H}$ is held fixed at cluster value.

b Statistics low, $C$-statistic used and goodness reported.

c Too few counts $(<10)$ detected for any spectral fitting; see the text.

${ }^{\mathrm{d}}$ Including an $\mathrm{Fe}$ line at $6.6 \mathrm{keV}$, assumed width $0.1 \mathrm{keV}$; see the text for details.

The light curve from the readout streak events shows a clear decline during the Chandra observation by a factor of $\sim 40 \%$. Power spectra from the readout streak events show a clear periodicity at $1000.0 \mathrm{~s}$ and its harmonics, identical to one of the spacecraft dither frequencies and thus a likely artifact (it is not seen in Rossi $X$-ray Timing Explorer (RXTE) data). No other periodicities (such as the 57-minute orbital period, Altamirano et al. 2010a) were identified, suggesting that the transient is not seen at high inclination.

\subsection{RXTE}

The RXTE has conducted regular scans of the Galactic bulge region since 2000 (generally covering NGC 6440 twice/ week), in part to search for faint transient sources below the sensitivity of RXTE's all-sky monitor (Swank \& Markwardt 2001), with results promptly made available. ${ }^{11}$ The PCA scans are significantly more sensitive in the Galactic bulge than the $R X T E$ all-sky monitor data. (Although dwell-by-dwell all-sky monitor data suggest a few points at $L_{X} \sim 10^{38} \mathrm{ergs} \mathrm{s}^{-1}$ from NGC 6440, suggesting X-ray bursts, their $6 \sigma$ maximum significance is matched by similar points from black hole candidates in the Galactic center. Thus, we cannot be assured of their reality.) Scans in late 2009 May showed evidence for an increased count rate from NGC 6440 (see the third red line in Figure 3). An outburst was confirmed by Swift observations in early June (see below). No further activity was observed until a scan showed a $5 \sigma$ detection on 2009 July 28 , near the beginning

\footnotetext{
11 http://lheawww.gsfc.nasa.gov/users/craigm/galscan/main.html
} 


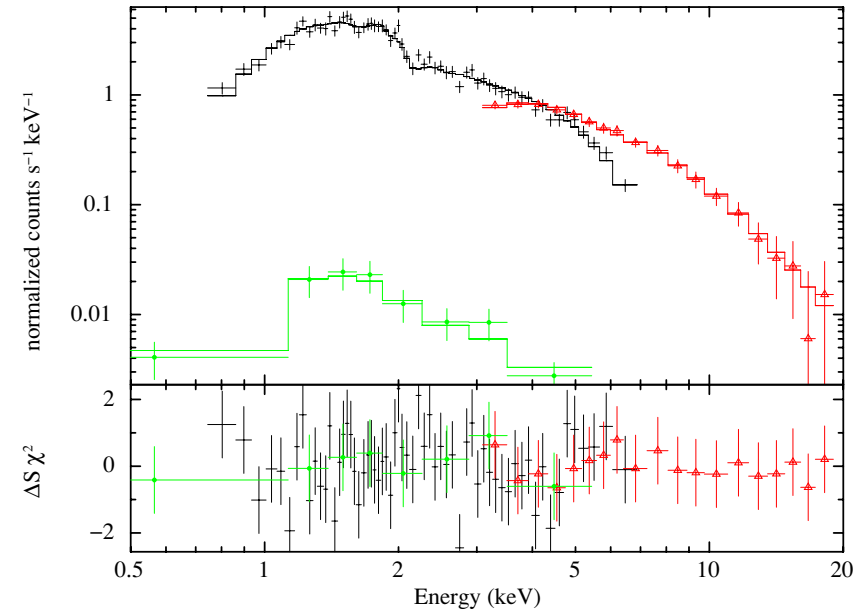

Figure 2. Spectra of NGC 6440 X-2 during the July/August outburst as observed by Chandra/ACIS-S (black online), RXTE/PCA (triangles; red online), and Swift/XRT (filled circles; green online). Top: data and absorbed power-law models. Bottom: residuals to fits. See Table 1 for details of spectral fits.

(A color version of this figure is available in the online journal.)

of the Chandra observation. A pointed RXTE observation on July 30 showed a significant decline from the Chandra and bulge scan fluxes (details below). Later bulge scans (on August 1 and 2) were consistent with a decay below the PCA's (backgroundlimited, due to its non-imaging nature) sensitivity (Figure 3).

A third bulge scan peak on 2009 August 29 triggered a pointed observation on August 30, which discovered millisecond pulsations from NGC $6440 \mathrm{X}-2$ with a frequency of $205 \mathrm{~Hz}$ (Altamirano et al. 2010a). Further RXTE observations starting on September 1 found fluxes returning to quiescence. Upon identifying a fourth outburst with Swift/XRT on 2009 October 1, a pointed $R X T E$ observation on October 2 detected NGC 6440 X-2 near the detection limit ( $\sim 1.5 \mathrm{mCrab})$. Altamirano et al. (2010a) report the detection of pulsations at a $3.4 \sigma$ level, further confirming the identification of this transient with NGC 6440 $\mathrm{X}$-2. RXTE observations on October 3 found it below the PCA's sensitivity limit. A fifth outburst was caught by $R X T E / P C A$ on October 28, returning to quiescence by November 1 . Below, we describe spectral analysis of these data; all $R X T E / P C A$ timing analysis is described in Altamirano et al. (2010a).

We extracted spectra from the PCA (Jahoda et al. 2006) detectors (PCU2 was consistently on, sometimes joined by other PCUs), excluding times when elevation angle ELV $<10^{\circ}$, source offset $>0.02$, or the time since the last South Atlantic Anomaly passage was $<30$ minutes. The background was modeled using the latest faint background model pca_bkgd_cmfaintl7_eMv20051128.mdl and the recorded times of South Atlantic Anomaly passage. ${ }^{12}$ Response files were created with the PCARMF (v. 11.1) tool. For spectral fitting, we exclude data below $3 \mathrm{keV}$ and above where the source can no longer be detected above our modeled background; this cut ranged between 10 and $30 \mathrm{keV}$, depending on the observation. HEXTE (cluster B, sensitive to the 20-200 keV energy range) did not detect the source even in the brightest (August 30) pointed $R X T E$ observation. $N_{H}$ was fixed to the cluster value due to $R X T E$ 's relative insensitivity to $N_{H}$. All our reported $L_{X}$ values are for $0.5-10 \mathrm{keV}$ (for consistency with other instruments; this extrapolation may incur additional systematic errors) assuming a distance of $8.5 \mathrm{kpc}$.

\footnotetext{
12 http://heasarc.gsfc.nasa.gov/docs/xte/pca_news.html
}

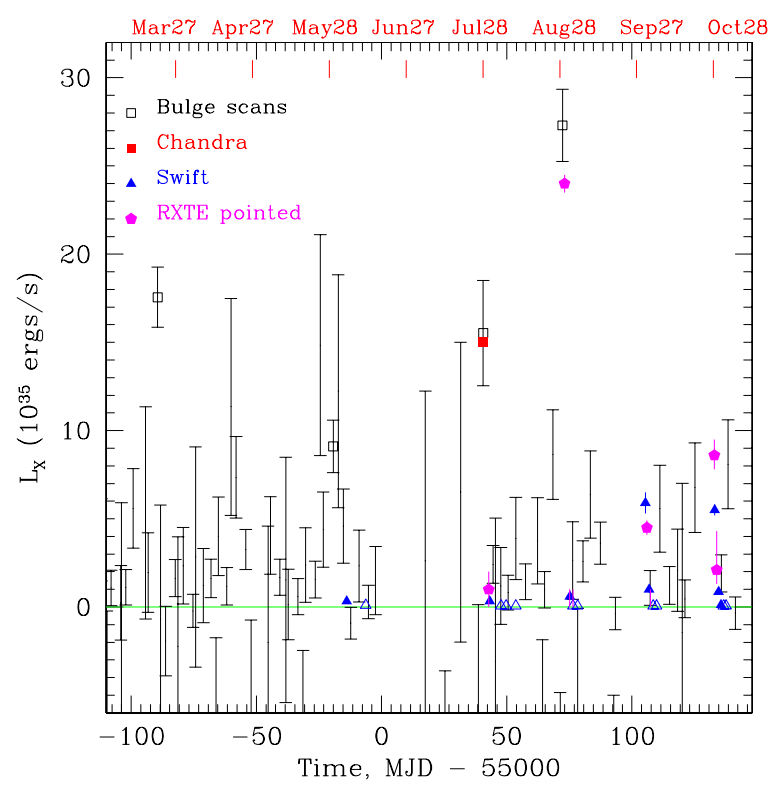

Figure 3. X-ray light curve of NGC 6440 over six months, with linear vertical scale. $R X T E / \mathrm{PCA}$ bulge scans are in black, with a box if they are $>4 \sigma$ above zero. RXTE/PCA-pointed observations are magenta pentagons. Swift observations are blue triangles (open if upper limits). The outburst Chandra observation is a red filled box. Red marks and dates at the top indicate the suggested 30.7-day recurrence epochs, which coincide with five clear episodes of X-ray activity.

(A color version of this figure is available in the online journal.)

The July 30 pointed observation could be fit with an absorbed power law with photon index $2.2 \pm 0.1$ and a luminosity of $(5 \pm 1) \times 10^{35} \mathrm{erg} \mathrm{s}^{-1}, 3.5$ times lower than the July 28 Chandra observation (Table 1; but see below). The August 30 observation showed a photon index of $1.84 \pm 0.02$ and $L_{X}=2.9 \times 10^{36} \mathrm{erg} \mathrm{s}{ }^{-1}$. The series of observations on September 1 and 2 showed much lower fluxes, averaging $L_{X}=4 \times 10^{35} \mathrm{erg} \mathrm{s}^{-1}$; the longest one also shows clear evidence of an iron line at $6.7 \pm 0.2 \mathrm{keV}$. However, simultaneous Swift observations (see below) find much lower fluxes from the cluster of $6 \times 10^{34} \mathrm{erg} \mathrm{s}^{-1}$, consistent with the quiescent cluster emission and inconsistent with the transient position. Therefore, we attribute the $R X T E / P C A$ flux observed on September 1 to the Galactic Ridge emission at this location. Subtracting this flux from the July $30 \mathrm{RXTE} / \mathrm{PCA}$ measurement gives $L_{X}=(1 \pm 1) \times 10^{35} \mathrm{erg} \mathrm{s}^{-1}$, a very marginal detection, and makes its spectral parameters unreliable.

We refine our spectral fitting of the August 30 observation by extracting spectra from only the top layer of PCU2 and modeling the inferred Galactic Ridge emission using our fits to the August 1 data. Fitting with an absorbed power law gives a good reduced chi-squared, but a residual near $6.5 \mathrm{keV}$ suggests the addition of an iron line (this is in addition to the Galactic Ridge iron line seen in the September data). Freezing $N_{H}$ to the cluster value, we obtain $\Gamma=1.78 \pm 0.03, L_{X}(0.5-10 \mathrm{keV})=$ $(2.4 \pm 0.1) \times 10^{36} \mathrm{erg} \mathrm{s}^{-1}$, with an iron line at $6.6_{-0.4}^{+0.6} \mathrm{keV}$, of equivalent width $54 \pm 39 \mathrm{eV}$ (90\% confidence). An $F$-test suggests that this is reasonable, providing $3.6 \%$ probability of such a $\Delta \chi^{2}$ by chance. Other AMXPs have displayed evidence of Fe K lines (e.g., SAX J1808.4-3658, Cackett et al. 2009; Papitto et al. 2009; HETE J1900.1-2455, Cackett et al. 2010; Swift J1756.9-2508, Patruno et al. 2010a), often showing relativistic broadening which our observations are unable to resolve.

Two $R X T E / P C A$ observations were obtained during the early October outburst, both during the source's decline. We identified 
pulsations in the first (October 1) observation, confirming that pulsations are common from this object (Altamirano et al. 2010a). The second (October 3) occurred after NGC 6440 X-2 had dipped below the PCA's sensitivity limit. We model the Galactic Ridge contribution to the October 1 observation, using the October 3 observation, finding $\Gamma=1.78 \pm 0.15, L_{X}(0.5-$ $10)=8.4_{-0.5}^{+0.8} \times 10^{35} \mathrm{erg} \mathrm{s}^{-1}$ (see Table 1$)$.

Three $R X T E /$ PCA observations were able to detect the October/November outburst. The first (on October 28) identified pulsations again (Altamirano et al. 2010a), at $L_{X}(0.5-$ $10 \mathrm{keV})=8.6_{-0.8}^{+0.9} \times 10^{35} \mathrm{ergs} \mathrm{s}^{-1}$ (see Table 1$)$, after subtraction of Galactic Ridge emission. RXTE/PCA observations detected emission over background on October 29 and (marginally) October 30, and observations on October 31 and after were consistent with Galactic Ridge emission.

The full RXTE/PCA Galactic bulge scan data reveals seven times from 2000 to 2009 when NGC 6440's count rates are $4 \sigma$ above zero. The two brightest outbursts $\left(L_{X}>10^{37} \mathrm{erg} \mathrm{s}^{-1}\right)$ have been identified with the other transient in NGC 6440, SAX J1748.9-2021 = CX1 (Altamirano et al. 2008; in't Zand et al. 2001). The other five potential outbursts are much fainter and briefer (only a single bulge scan point each, so lasting less than a week), of which three were discussed above. The other two are 2007 April 15 and 2009 March 20. A period of 30.7 days $( \pm 0.3$ days, from the uncertainties in the peak of the late October outburst), with reference date MJD 55040.5, reasonably predicts the peaks of the four well-studied outbursts. The model predicts missed outbursts on April 27 (weak activity is suggested by bulge scans on April 18-20), May 28 (a bulge scan detection occurred on May 25, followed by a faint Swift detection on June 4), and June 27 (no bulge scans conducted \pm 10 days around this date). The bulge scan point in March is off the prediction by $7 \pm 1$ days, indicating either that the outburst period has slightly decreased over time (as the April and May evidence also suggest) or that the March point is not a real outburst. Sensitive monitoring since November 2 (up to 2010 February 11) has not been possible due to solar constraints and an outburst of SAX J1748.9-2021 (Suzuki et al. 2010; Patruno et al. 2010b). We show the bulge scan data in the relevant date range (and detections and upper limits from Swift and Chandra) in Figure 3; see also Altamirano et al. (2010a). We use an absorbed power law of photon index 2 to convert $R X T E / P C A$ bulge scan fluxes to $0.5-10 \mathrm{keV}$ unabsorbed fluxes with PIMMS ${ }^{13}$ and assume a distance of $8.5 \mathrm{kpc}$ to estimate luminosities.

\subsection{Swift}

Eighteen Swift/XRT observations were performed, tracking five outbursts from NGC 6440 X-2 (see Table 1). We extracted all Swift XRT spectra from 20 pixel radii (except for October 1 and 28, see below) and background from a surrounding annulus. We downloaded the response matrix swxpc0to12_20010101v009.rmf from the Swift Web site ${ }^{14}$ and created effective area files using the XRTMKARF tool. Spectra with more than 50 counts were binned with 15 counts/bin for $\chi^{2}$ statistics, those with fewer used C-statistics (either binned with 5 counts/bin or unbinned), while we produced only luminosity limits for less than 10 detected counts.

A Swift X-ray Telescope (XRT) observation on 2009 June 4 found enhanced X-ray emission from NGC 6440. Using the

\footnotetext{
13 http://asc.harvard.edu/toolkit/pimms.jsp

14 http://swift.gsfc.nasa.gov
}

FTOOL XRTCENTROID on the June 4 XRT source, we identified a position of R.A. $=17: 48: 52.73$, decl. $=-20: 21: 24.1$ with an error radius of $5^{\prime \prime}$. This position is consistent with NGC 6440 X-2 (see Figures 1 and 4), but not with other known X-ray sources in NGC 6440, so we conclude it is the same source. A second Swift observation on June 11 found much weaker emission, at position R.A. = 17:48:52.64, decl. $=-20: 21: 29.9$, with error radius 7 .'3, consistent with either NGC 6440 X-2 or with the cluster center. Spectral fitting of the few detected photons with an absorbed power law derives a photon index of $4.2_{-1.1}^{+1.2}$ and $L_{X}=1.0_{-0.5}^{+1.0} \times 10^{34} \mathrm{erg} \mathrm{s}^{-1}$. This is consistent with emission from the rest of the cluster, containing a mixture of soft quiescent LMXBs and sources (likely cataclysmic variables) with harder spectra (Pooley et al. 2002; Heinke et al. 2003).

We obtained four Swift observations soon after the initial Chandra discovery (Table 1). On July 31, Swift/XRT found enhanced emission from NGC 6440 X-2's position. Observations on August 4, 6, and 10 found fluxes and positions consistent with the cluster center, and soft spectra (Table 1, Figures 4 and 5). The centroid of the emission in the deepest of these observations (August 6, $1.9 \mathrm{ks}$ ) is R.A. = 17:48:52 $\mathrm{s} .9$, decl. $=-20: 21: 35.1$, with an error radius of 6 .' 1 , which is consistent with the cluster center but not with NGC 6440 X-2.

After the August 29 bulge scan detection, Swift observed NGC 6440 on September 1, identifying emission from the location (R.A. $=17: 48: 52.6$, decl. $=-20: 21: 24.9$, error radius 4 ." 8$)$ of NGC 6440 X-2 at $L_{X}=(6 \pm 2) \times 10^{34} \mathrm{erg} \mathrm{s}^{-1}$ (Figures 4 and 5). Further Swift observations on September 2 and 4 found faint emission, consistent in position and flux with the cluster.

Swift monitoring on October 1 found NGC 6440 X-2 back in outburst. The count rate was high enough to produce pileup (in photon counting mode); we fit the radial profile with a King model and identified a $6^{\prime \prime}$ core, so we extracted a spectrum from an annulus from $6^{\prime \prime}$ to $45^{\prime \prime}$ (20 pixels). On October 2, Swift identified a declining flux from NGC $6440 \mathrm{X}-2$, returning to quiescence by October 4 (Figures 4 and 5).

A Swift observation on October 29 confirmed that NGC 6440 $\mathrm{X}-2$ was back in outburst, and relatively bright; we dealt with pileup as described above. Observations on October 30 and 31 observed X-2's decay, which by November 1 was below Swift/ XRT's detection limit (Figures 4 and 5).

\subsection{Chandra in Quiescence}

We combined two Chandra ACIS-S observations of NGC 6440 (total exposure $48 \mathrm{ks}$ ) when no transients were in outburst (ObsIDs 947 and 3799; Pooley et al. 2002; Cackett et al. 2005) to look for evidence of NGC 6440 X-2's X-ray emission in quiescence. The observations were reprocessed and aligned, and filtered to produce a $0.3-7 \mathrm{keV}$ band image (Figure 1). No photons lie within a $1^{\prime \prime}$ circle around NGC $6440 \mathrm{X}-2$. Using 2.3 photons as our $90 \%$ confidence upper limit (Gehrels 1986), we compute limits on the unabsorbed quiescent luminosity. For a power law of index 2.2, $L_{X}(0.5-10 \mathrm{keV})<7.4 \times 10^{30} \mathrm{erg} \mathrm{s}^{-1}$, while for a hydrogen-atmosphere neutron star model (Heinke et al. 2006), $L_{X}(0.5-10 \mathrm{keV})<1.6 \times 10^{31} \mathrm{erg} \mathrm{s}^{-1}$ and $L_{\mathrm{NS}}$ $(0.01-10 \mathrm{keV})<6.0 \times 10^{31} \mathrm{erg} \mathrm{s}^{-1}$ (we use $L_{\mathrm{NS}}$ as the total emission from the NS surface).

We obtained a 5 ks ACIS-S follow-up Chandra Director's Discretionary Time observation on 2009 August 10, to see if NGC $6440 \mathrm{X}-2$ had returned to full quiescence, or was continuing to accrete at $L_{X}(0.5-10 \mathrm{keV}) \sim 5 \times 10^{32} \mathrm{erg} \mathrm{s}^{-1}$, similarly to SAX J1808.4-3658 at the end of its 2008 outburst 

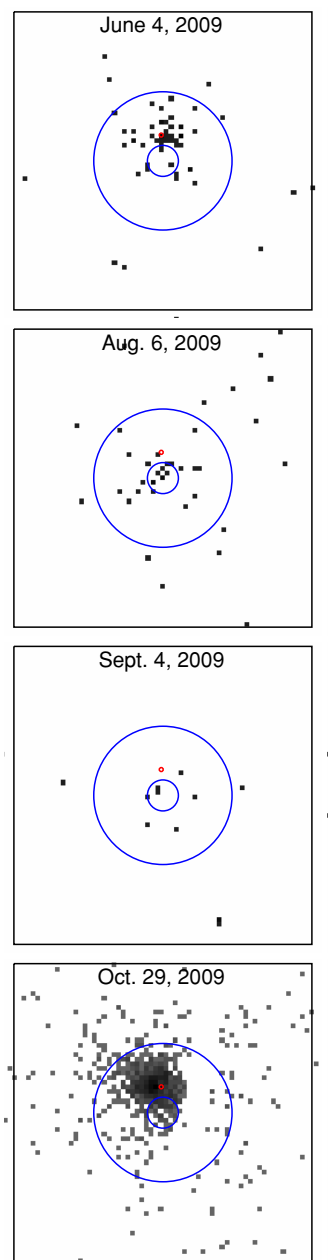
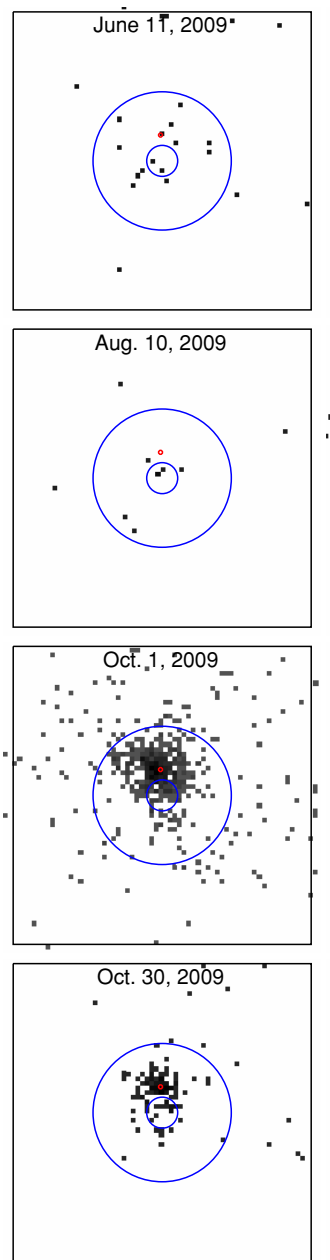
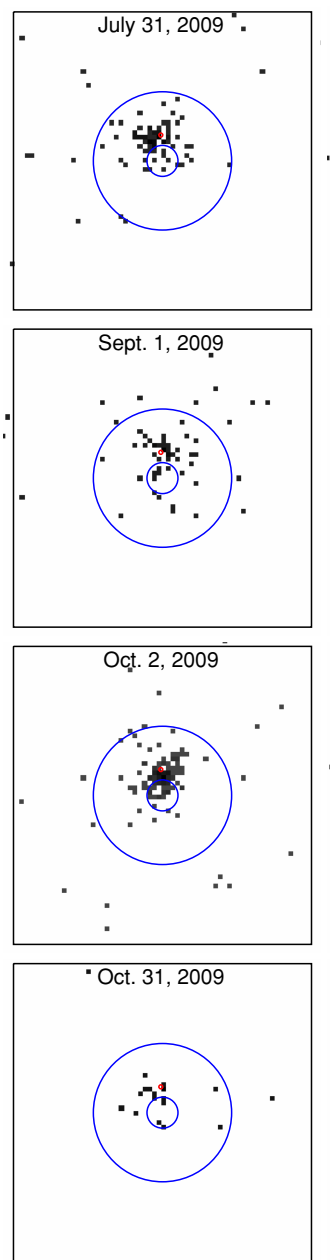
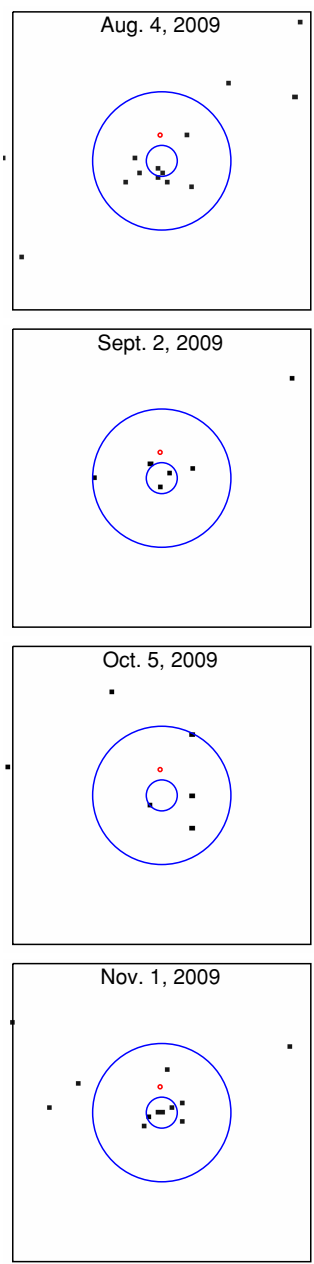

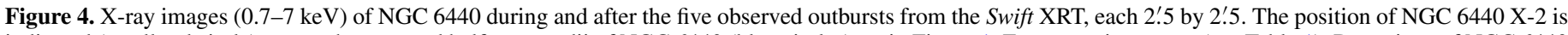

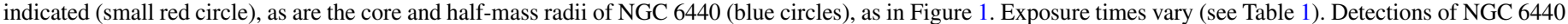
X-2 are seen on June 4, July 31, September 1, and October 1, 2, 29, 30, and 31; the remaining emission is attributable to the other cluster sources (see Figure 1).

(A color version of this figure is available in the online journal.)

(Campana et al. 2008). Two photons were detected at the position of NGC $6440 \mathrm{X}-2$ (see Figure 1). As the background is quite low $(<0.01$ photons expected), both photons are probably from NGC 6440 X-2. We estimate (for an assumed spectrum similar to that in outburst) $L_{X}=6_{-4}^{+9} \times 10^{31} \mathrm{erg} \mathrm{s}^{-1}$, with uncertainties from Gehrels (1986) at $90 \%$ confidence. This confirms that NGC $6440 \mathrm{X}-2$ returned to quiescence, although it appears brighter than the limits from the 2000 and 2003 quiescent observations (above).

\section{OPTICAL/INFRARED OBSERVATIONS}

\subsection{Archival NTT and HST}

The location of NGC 6440 X-2 has been previously observed by the European Southern Observatory's New Technology Telescope (NTT) and by the Hubble Space Telescope (HST) (Piotto et al. 2002). The NTT imaging in $R$ and $B$ was described by Verbunt et al. (2000), and the $H S T$ imaging in $V$ and $B$ was described by Piotto et al. (2002). We identified UCAC2 standards to place the NTT $R$ astrometric frame onto the ICRS frame, and then by identifying common stars in the WFPC2 and NTT frames, to place the HST astrometric frame onto the ICRS frame, with an uncertainty of $0.2(1 \sigma)$. No star can be identified within $4 \sigma$ of the transient position on the
NTT and WFPC2 $B$ frames, but one star in the WFPC2 $V$ frame is located 0.'4 from the transient position (Figure 6). Calibrating our photometry with that in Piotto et al. (2002), we find a magnitude of $V=21.0 \pm 0.2$ for this object. Based on objects in the HST image that are just barely detected in $B$, we estimate $B>22.0$. These images were probably taken during quiescence, and NGC 6440 X-2's 57 minute orbital period indicates that it will be very faint. As the ultracompact LMXB XTE J0929-314 in quiescence may have been detected with $M_{V}=13.2$ (D'Avanzo et al. 2009; thus it would have $V=31$ in NGC 6440), we think it unlikely that this star is the true counterpart.

\subsection{Outburst and Decay}

Li et al. (2009) reported that unfiltered images were taken of NGC 6440 on 2009 July 30 and 31 with the 0.76 m Katzman Automatic Imaging Telescope (KAIT). Their image subtraction against previous KAIT observations $(2007,2008)$ revealed no evidence of an optical transient, with limiting magnitudes of 19.5-20.0.

We obtained images with the CTIO 4-meter telescope using the ISPI infrared imager on 2009 August $2-4$ in the $J$ and $K$ bands (Figure 7). The total exposure times were 12 and 8 minutes per night, giving theoretical magnitude limits of 19.5 and 18 in $J$ and 

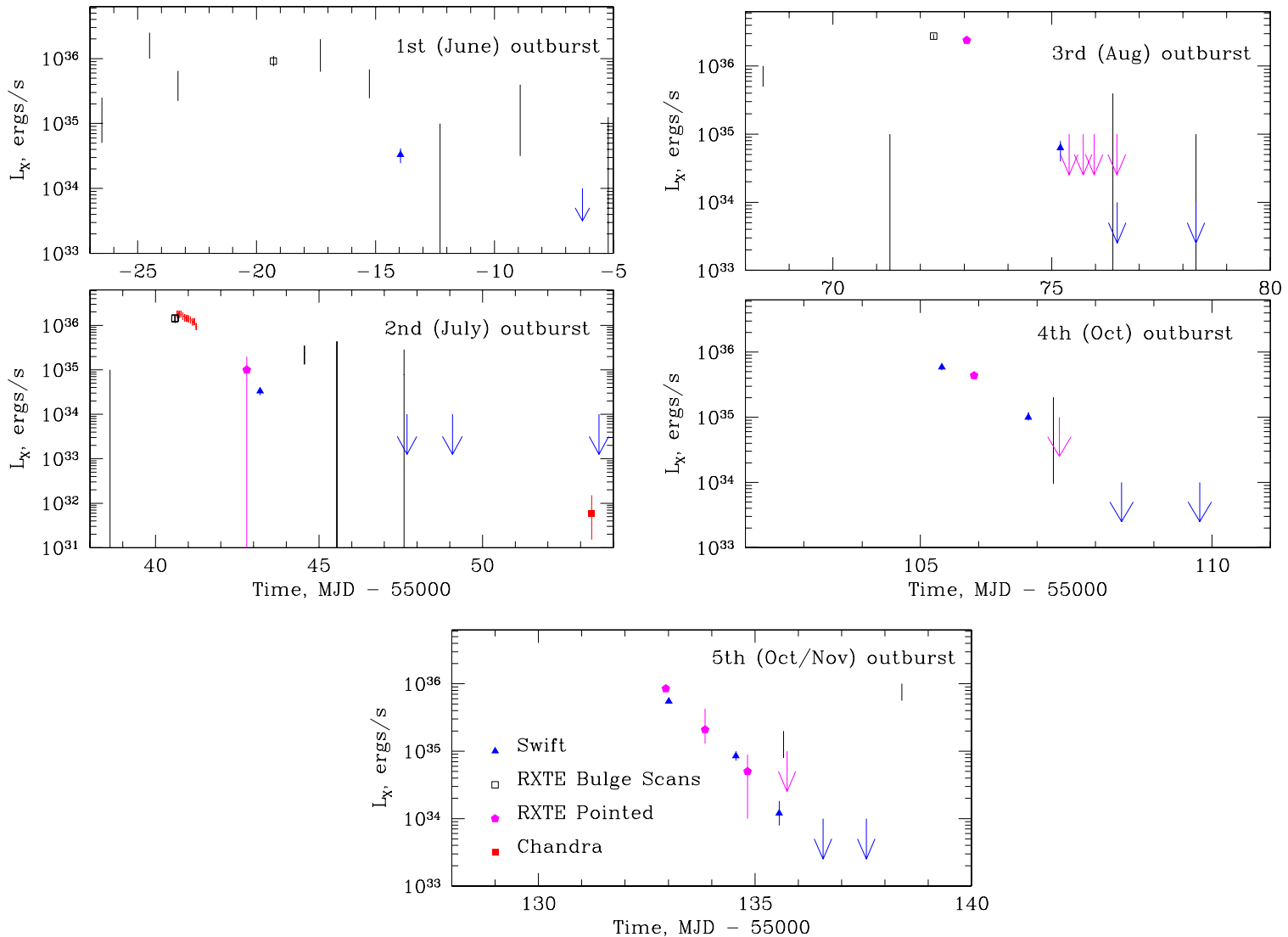

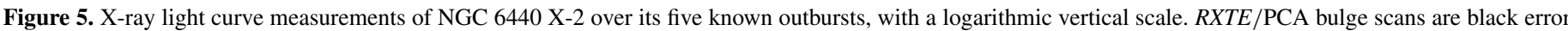

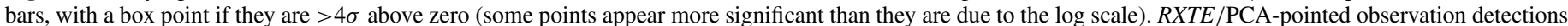

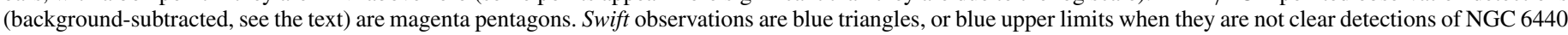
X-2. Chandra observations are the red-filled box and series of red crosses (showing the light curve from the June observation).

(A color version of this figure is available in the online journal.)
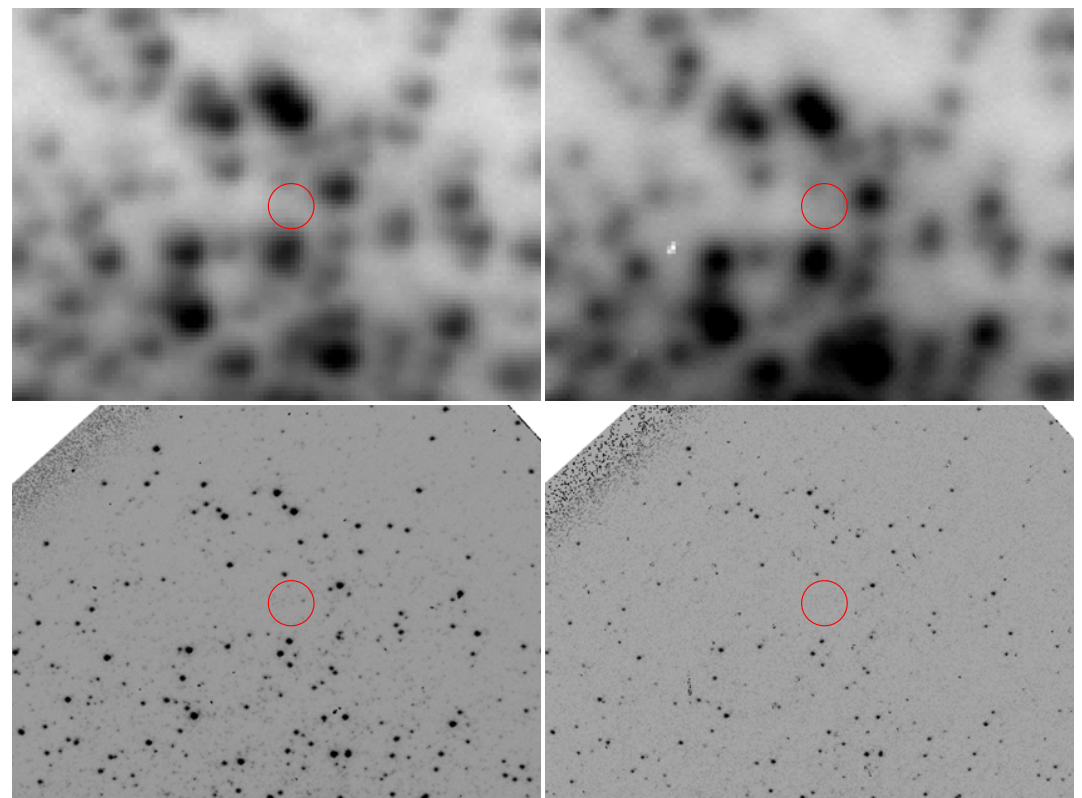

Figure 6. Optical images of NGC 6440, plotting an 0'8 $(4 \sigma)$ error circle for NGC 6440 X-2. Upper left: archival NTT $R$ frame; upper right: new Gemini $g^{\prime}$ frame; lower left: HST WFPC2 $V$ frame; lower right: $H S T$ WFPC2 B frame. All $19^{\prime \prime} \times 14^{\prime \prime}$, with N at top.

(A color version of this figure is available in the online journal.)

$K$, respectively (with a signal-to-noise ratio of 10 ). The airmass was around 1.1 and the seeing was $1^{\prime \prime}$ the first night, increasing to $1^{\prime \prime} .5$ the last night. The images have been taken using a dithered pattern and an offset blank field has been observed to estimate the sky contribution to the emission. We used IRAF common packages and the PANIC package (Martini et al. 2004) 


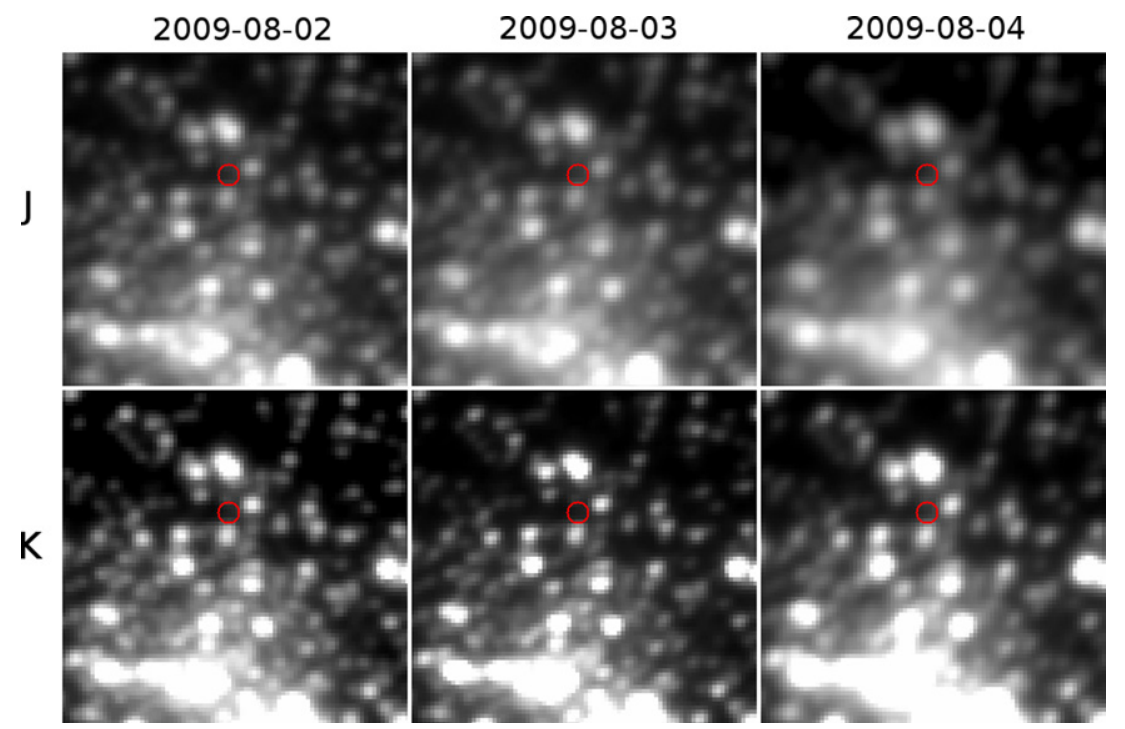

Figure 7. Infrared ISPI images (each $25^{\prime \prime} \times 25^{\prime \prime}, \mathrm{N}$ at top) with the CTIO $4 \mathrm{~m}$ on the dates specified. Top: images in $J$; bottom: images in $K$. Circles ( 0 "' 8 in radius) indicate the positions of NGC $6440 \mathrm{X}-2$.

(A color version of this figure is available in the online journal.)

to reduce the data and then SExtractor/Scamp (Bertin 2006) to calibrate the astrometry and photometry of the image, using the 2MASS catalog as a reference (Skrutskie et al. 2006). Based on the detection of sources around NGC $6440 \mathrm{X}-2$, a source of magnitude $K=17$ and $J=18.5$ would have been detected.

We obtained three $500 \mathrm{~s}$ frames in each of $g^{\prime}$ (note that the $g^{\prime}$ bandpass is between $V$ and $B$ ) and $r^{\prime}$ using GeminiSouth GMOS-S on 2009 September 1, during the outburst decay (program GS-2009B-DD-2). Unfortunately the $r^{\prime}$ frames were saturated at the location of the transient, but the $g^{\prime}$ frames provided our best optical outburst limit (Figure 6). No star was seen within 0 .'8 $(4 \sigma)$ of the transient position. An approximate calibration of the $g^{\prime}$ images was obtained using a star-by-star comparison with the $B, V$ photometry of Martins et al. (1980). We determined the detection limit for stars within an annulus about the cluster center containing the location of the transient. Based on the faintest $5 \%$ of the stars in the annulus, the estimated detection limit is $g^{\prime} \sim 22.0$. Thus, we adopt $g^{\prime}>22$ as an approximate limit for NGC $6440 \mathrm{X}-2$, at a time when Swift found its X-ray luminosity to be $6 \times 10^{34} \mathrm{erg} \mathrm{s}^{-1}$.

\section{DISCUSSION}

The discovery of millisecond pulsations (Altamirano et al. 2010a) clearly identifies NGC $6440 \mathrm{X}-2$ as a neutron star LMXB system and provides the orbital period of 57.3 minutes. The ultracompact nature of NGC 6440 X-2 confirms the trend (Deutsch et al. 2000) for globular clusters to have a higher fraction of ultracompact LMXBs than field systems, indicating a different formation mechanism (Ivanova et al. 2005). However, it goes against the apparent trend (Zurek et al. 2009) for ultracompacts in globular clusters to have shorter periods than ultracompacts in the field. The lack of optical detections is not surprising, when the ultracompact orbit is considered. Using the relation between absolute magnitude, orbital period, and X-ray luminosity derived by van Paradijs \& McClintock (1994), we predict $M_{V}=3.8$ and thus $V=21.8$ at peak. The scatter in this relation is about 1.5 magnitudes and our optical observations all occurred during the outburst decays, so our limits on NGC 6440 $\mathrm{X}-2$ are consistent with this relation.
NGC 6440 X-2's outbursts differ from those of other globular cluster LMXBs, both in the faintness of X-2's outbursts (peak $0.5-10 \mathrm{keV}, L_{X}=1.5 \times 10^{36} \mathrm{erg} \mathrm{s}^{-1}$ for the July outburst, $2.8 \times 10^{36} \mathrm{erg} \mathrm{s}^{-1}$ for the August outburst), and in their brevity. The July X-ray light curve indicates that the time spent above $10^{35} \mathrm{erg} \mathrm{s}^{-1}$ was no more than 4 days, and perhaps only 2.5 days (Figure 5), one of the shortest transient LMXB outbursts so far recorded (cf. Natalucci et al. 2000; in't Zand et al. 2004; Wijnands et al. 2009). The August outburst light curve indicates $<3.5$ days spent above $10^{35} \mathrm{erg} \mathrm{s}^{-1}$, and the light curves from the other outbursts, while less constraining, are consistent with this timescale (Figure 5). We note that another ultracompact AMXP, XTE J1751-305, has similarly shown short and faint outbursts (Markwardt et al. 2007, 2009; Linares et al. 2007).

Why does NGC 6440 X-2 show such faint and brief outbursts? The obvious drivers are accretion disk instabilities or magnetospheric instabilities. However, magnetospheric instabilities occur on much faster timescales (e.g., the Rapid Burster; Lewin 1993) and would require that the disk remains viscous (and thus ionized) between outbursts, which seems unlikely. Standard accretion disk instability models (e.g., Lasota 2001) predict quiescent periods 10 times longer, and brighter and longer outbursts, for systems with 57 minute orbital periods. Naively, we expect longer intervals between outbursts for a 57 minute system than for the other known ultracompact systems, if NGC 6440 X-2 is indeed an evolutionary descendant of systems like them, as it will have a larger disk and lower mass transfer rate (e.g., Deloye \& Bildsten 2003). However, we are not aware of detailed modeling of outbursts of hydrogen-poor accretion disks with extreme mass ratios (and thus likely superhumps, Whitehurst 1988), suggesting an avenue for further study.

The X-ray record suggests that NGC 6440 X-2's activity has significantly increased in the past year. It would be difficult to attribute this change to the disk (as it includes numerous outburst cycles), and we suggest that it represents a signal of mass transfer variations from the donor. Cyclical variations in the orbital period are well established in longer-period cataclysmic variables (Borges et al. 2008), LMXBs (Wolff et al. 2009), and black widow pulsars (Arzoumanian et al. 1994) and seem likely to be due to magnetic activity in 


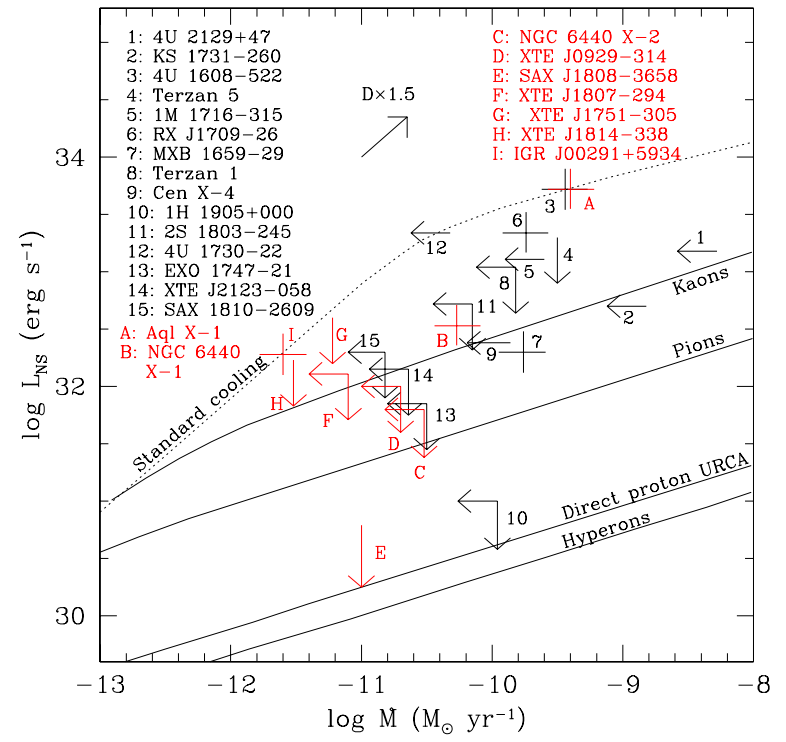

Figure 8. Measurements of, or limits on, the quiescent thermal luminosity of various NS transients, compared to estimates of, or upper limits on, their time-averaged mass accretion rates. Data from compilations of Heinke et al. (2007, 2009b), with NGC 6440 X-2 added. Predictions of standard cooling and several enhanced cooling mechanisms are plotted, following Yakovlev \& Pethick (2004). Accreting millisecond pulsars are indicated separately (in red), while the effect of increasing the distance by a factor of 1.5 for any system is indicated with an arrow labeled " $\mathrm{D} \times 1.5$."

(A color version of this figure is available in the online journal.)

the companion star. Such activity has also been suggested to explain SAX J1808.4-3658's large current rate of orbital period increase (Hartman et al. 2008) and decades-long variations in mass transfer rates in LMXBs (Durant et al. 2010). If such a mechanism is active here, it requires a partly nondegenerate, convective companion. Alternatively, the increased activity could indicate a change in the orbital parameters, induced by a distant companion (making this a triple system; testable with monitoring of future outbursts by $R X T E$ ) or a recent close interaction with another star (its position outside the core suggests that this is less likely).

It is difficult to estimate NGC $6440 \mathrm{X}$-2's mass transfer rate due to its extreme faintness and the limitations of existing surveys. We identify two limiting cases: one based on its recent outburst history and the other using the full bulge scan light curve. For the first case, we estimate the outbursts as lasting 3 days at an average $L_{X} \sim 1.5 \times 10^{36} \mathrm{erg} \mathrm{s}^{-1}$, and occurring every 31 days. For canonical neutron star mass and radius estimates, this gives a time-averaged mass accretion rate of $3 \times 10^{-11} M_{\odot} \mathrm{yr}^{-1}$. Although this represents the mass transfer rate over the past few months, it is clear that NGC 6440 $\mathrm{X}-2$ has not shown such outbursts regularly over the entire bulge scan epoch, where only five outbursts have been identified. Assuming (generously) that two-thirds of all outbursts have been missed (the October and November outbursts were missed by bulge scans, and it seems likely that outbursts in June/July and April/May were missed; Figure 3), and that the average outburst is like those seen so far, we estimate NGC 6440 X-2's mass transfer rate over the entire bulge scan epoch (ten years) as $1.3 \times 10^{-12} M_{\odot} \mathrm{yr}^{-1}$. This latter rate is consistent with an ultracompact binary with an orbital period of 57 minutes experiencing conservative mass transfer driven by general relativistic angular momentum loss (Deloye \& Bildsten 2003), though a higher rate is not inconsistent with a relatively high-entropy (partly nondegenerate) donor.
The tight upper limit on NGC 6440 X-2's quiescent emission is the third lowest for any neutron star LMXB, after the transients SAX J1808.4-3658 and 1H 1905+000 (Heinke et al. 2009b; Jonker et al. 2007, Figure 8). Deep Chandra observations might substantially improve these limits (e.g., $100 \mathrm{ks}$ could reduce the quiescent flux limit by a factor of 3). Long-term study of outbursts from this system will allow a better measure of the average mass accretion rate. It will be of great interest to see if the outbursts continue to occur every $\sim 31$ days, turn off, or change their outburst frequency, as this system's behavior is extremely unusual.

This is the first globular cluster to show two transiently outbursting X-ray sources. Many candidate quiescent LMXBs have been identified in globular clusters through their soft spectra, including eight in NGC 6440 (Grindlay et al. 2001; Rutledge et al. 2002; Pooley et al. 2002; Heinke et al. 2003), although few have been observed to undergo outbursts. Some of these quiescent LMXBs may be producing short, faint transient outbursts like NGC 6440 X-2's, which are at or near the noise level for existing surveys such as the RXTE/PCA bulge scans and all-sky monitor. Even fainter X-ray transients have been studied in the Galactic center with dedicated observations (Muno et al. 2005; Wijnands et al. 2006). Swift could efficiently survey one or a few of the globular clusters richest in quiescent LMXBs for such small-scale outbursts.

We are grateful to N. Gehrels and the Swift team, H. Tananbaum and the Chandra team, M. Pretorius at ESO, M. Buxton at SMARTS, the RXTE team, N. Levenson, J. Radomski, R. Carrasco, and the Gemini-South science team, for rapidly scheduling observations of NGC 6440. We thank D. Pooley, S. Ransom, N. Degenaar, and A. Kong for discussions and the referee for a useful, clear, and rapid report. This research has made use of data obtained through the High Energy Astrophysics Science Archive Research Center (online service), provided by the NASA/Goddard Space Flight Center. We acknowledge the use of public data from the Swift, RXTE, Chandra, HST, and ESO data archives.

Facilities: RXTE (PCA), CXO (ACIS), Swift (XRT), Gemini:South (GMOS), HST (WFPC2), NTT, Blanco (ISPI IR Imager)

Note added in proof. As this paper went to press, NGC 6440 X-2 was detected again in outburst by Swift and RXTE, on 2010 March 19-21 (Altamirano et al. 2010b), confirming that its outbursts are continuing.

\section{REFERENCES}

Altamirano, D., Casella, P., Patruno, A., Wijnands, R., \& van der Klis, M. 2008, ApJ, 674, L45

Altamirano, D., Patruno, A., Heinke, C., Linares, M., Markwardt, C., \& Strohmayer, T. 2010b, ATel, 2500

Altamirano, D., et al. 2009, ATel, 2182

Altamirano, D., et al. 2010a, ApJ, 712, L58

Arzoumanian, Z., Fruchter, A. S., \& Taylor, J. H. 1994, ApJ, 426, L85

Bertin, E. 2006, in ASP Conf. Ser. 351, Astronomical Data Analysis Software and Systems XV, ed. C. Gabriel et al. (San Francisco, CA: ASP), 112

Borges, B. W., Baptista, R., Papadimitriou, C., \& Giannakis, O. 2008, A\&A, 480, 481

Cackett, E. M., et al. 2005, ApJ, 620, 922

Cackett, E. M., et al. 2009, ApJ, 694, L21

Cackett, E. M., et al. 2010, ApJ, submitted (arXiv:0908.1098)

Campana, S., Stella, L., \& Kennea, J. A. 2008, ApJ, 684, L99

Clark, G. W. 1975, ApJ, 199, L143

D’Avanzo, P., Campana, S., Casares, J., Covino, S., Israel, G. L., \& Stella, L. 2009, A\&A, 508, 297

Deloye, C. J., \& Bildsten, L. 2003, ApJ, 598, 1217 
Deutsch, E. W., Margon, B., \& Anderson, S. F. 2000, ApJ, 530, L21

Di Stefano, R., Kong, A. K. H., Garcia, M. R., Barmby, P., Greiner, J., Murray, S. S., \& Primini, F. A. 2002, ApJ, 570, 618

Dotani, T., Inoue, H., Murakami, T., Nagase, F., \& Tanaka, Y. 1990, Nature, 347,534

Durant, M., Cornelisse, R., Remillard, R., \& Levine, A. 2010, MNRAS, 401, 355

Gavriil, F. P., Strohmayer, T. E., Swank, J. H., \& Markwardt, C. B. 2007, ApJ, 669, L29

Gehrels, N. 1986, ApJ, 303, 336

Grindlay, J. E., Heinke, C., Edmonds, P. D., \& Murray, S. S. 2001, Science, 292, 2290

Harris, W. E. 1996, AJ, 112, 1487

Hartman, J. M., et al. 2008, ApJ, 675, 1468

Heinke, C. O., Altamirano, D., \& Markwardt, C. 2009a, ATel, 2180

Heinke, C. O., \& Budac, S. A. 2009, ATel, 2139

Heinke, C. O., Grindlay, J. E., Lugger, P. M., Cohn, H. N., Edmonds, P. D., Lloyd, D. A., \& Cool, A. M. 2003, ApJ, 598, 501

Heinke, C. O., Jonker, P. G., Wijnands, R., Deloye, C. J., \& Taam, R. E. 2009b, ApJ, 691, 1035

Heinke, C. O., Jonker, P. G., Wijnands, R., \& Taam, R. E. 2007, ApJ, 660, 1424

Heinke, C. O., Rybicki, G. B., Narayan, R., \& Grindlay, J. E. 2006, ApJ, 644, 1090

Heinke, C. O., et al. 2009c, ATel, 2143

Hertz, P., \& Grindlay, J. E. 1983, ApJ, 275, 105

Homer, L., Deutsch, E. W., Anderson, S. F., \& Margon, B. 2001, AJ, 122, 2627

Hut, P., Murphy, B. W., \& Verbunt, F. 1991, A\&A, 241, 137

in't Zand, J. J. M., van Kerkwijk, M. H., Pooley, D., Verbunt, F., Wijnands, R., \& Lewin, W. H. G. 2001, ApJ, 563, L41

in't Zand, J. J. M., et al. 1999, A\&A, 345, 100

in't Zand, J., et al. 2004, Nucl. Phys. B, 132, 486

Ivanova, N., Rasio, F. A., Lombardi, J. C., Jr., Dooley, K. L., \& Proulx, Z. F. 2005, ApJ, 621, L109

Jahoda, K., et al. 2006, ApJS, 163, 401

Jonker, P. G., Steeghs, D., Chakrabarty, D., \& Juett, A. M. 2007, ApJ, 665, L147

Jordán, A., et al. 2004, ApJ, 613, 279

Kundu, A., Maccarone, T. J., \& Zepf, S. E. 2002, ApJ, 574, L5

Lasota, J. P. 2001, New Astron. Rev., 45, 449

Lewin, W. H. G. 1993, Space Sci. Rev., 62, 223

Li, W., Chornock, R., \& Filippenko, A. V. 2009, ATel, 2144

Linares, M., Wijnands, R., \& van der Klis, M. 2007, ATel, 1055

Maccarone, T. J., Kundu, A., Zepf, S. E., \& Rhode, K. L. 2007, Nature, 445, 183

Markert, T. H., Backman, D. E., Canizares, C. R., Clark, G. W., \& Levine, A. M. 1975, Nature, 257, 32
Markwardt, C. B., Altamirano, D., Strohmayer, T. E., \& Swank, J. H. 2009 ATel, 2237

Markwardt, C. B., Pereira, D., \& Swank, J. H. 2007, ATel, 1051

Markwardt, C. B., \& Swank, J. H. 2005, ATel, 495

Martini, P., Persson, S. E., Murphy, D. C., Birk, C., Shectman, S. A., Gunnels, S. M., \& Koch, E. 2004, Proc. SPIE, 5492, 1653

Martins, D. H., Harvel, C. A., \& Miller, D. H. 1980, AJ, 85, 521

Muno, M. P., Pfahl, E., Baganoff, F. K., Brandt, W. N., Ghez, A., Lu, J., \& Morris, M. R. 2005, ApJ, 622, L113

Natalucci, L., et al. 2000, ApJ, 536, 891

Ortolani, S., Barbuy, B., \& Bica, E. 1994, A\&AS, 108, 653

Papitto, A., di Salvo, T., Iaria, R., Burderi, L., Riggio, A., Menna, M. T., \& Robba, N. R. 2009, A\&A, 493, L39

Patruno, A., Altamirano, D., \& Messenger, C. 2010a, MNRAS, 403, 1426

Patruno, A., et al. 2010b, ATel, 2407

Piotto, G., et al. 2002, A\&A, 391, 945

Pooley, D., et al. 2002, ApJ, 573, 184

Pooley, D., et al. 2003, ApJ, 591, L131

Rutledge, R. E., Bildsten, L., Brown, E. F., Pavlov, G. G., \& Zavlin, V. E. 2002, ApJ, 578, 405

Sarazin, C. L., Kundu, A., Irwin, J. A., Sivakoff, G. R., Blanton, E. L., \& Randall, S. W. 2003, ApJ, 595, 743

Sivakoff, G. R., et al. 2007, ApJ, 660, 1246

Skrutskie, M. F., et al. 2006, AJ, 131, 1163

Suzuki, M., et al. 2010, ATel, 2360

Swank, J., \& Markwardt, K. 2001, in ASP Conf. Ser. 251, New Century of X-ray Astronomy, ed. H. Inoue \& H. Kunieda (San Francisco, CA: ASP), 94 van Paradijs, J., \& McClintock, J. E. 1994, A\&A, 290, 133

Verbunt, F. 2003, in ASP Conf. Ser. 296, New Horizons in Globular Cluster Astronomy, ed. G. Piotto et al. (San Francisco, CA: ASP), 245

Verbunt, F., \& Hut, P. 1987, in IAU Symp. 125, The Origin and Evolution of Neutron Stars, ed. D. J. Helfand \& J.-H. Huang (Dordrecht: Kluwer), 187

Verbunt, F., \& Lewin, W. H. G. 2006, in Compact Stellar X-Ray Sources, ed. W. Lewin \& M. van der Klis (Cambridge Astrophys. Ser. 39; Cambridge: Cambridge Univ. Press), 341

Verbunt, F., van Kerkwijk, M. H., in’t Zand, J. J. M., \& Heise, J. 2000, A\&A, 359,960

White, N. E., \& Angelini, L. 2001, ApJ, 561, L101

Whitehurst, R. 1988, MNRAS, 232, 35

Wijnands, R., Rol, E., Cackett, E. M., Starling, R. L. C., \& Remillard, R. A. 2009, MNRAS, 393, 126

Wijnands, R., et al. 2006, A\&A, 449, 1117

Wolff, M. T., Ray, P. S., Wood, K. S., \& Hertz, P. L. 2009, ApJS, 183, 156

Yakovlev, D. G., \& Pethick, C. J. 2004, ARA\&A , 42, 169

Zurek, D. R., Knigge, C., Maccarone, T. J., Dieball, A., \& Long, K. S. 2009, ApJ 699, 1113 